\title{
Completely Positive Quantum Dissipation
}

\author{
Bassano Vacchini \\ Dipartimento di Fisica dell'Università di Milano and INFN, Sezione di Milano, Via Celoria 16, I-20133 Milan, Italy \\ and Fachbereich Physik, Philipps-Universität, Renthof 7, D-35032 Marburg, Germany
}

(Received 9 April 1999)

\begin{abstract}
A completely positive master equation describing quantum dissipation for a Brownian particle is derived starting from microphysical collisions, exploiting a recently introduced approach to subdynamics of a macrosystem. The obtained equation can be cast into Lindblad form with a single generator for each Cartesian direction. Temperature dependent friction and diffusion coefficients for both position and momentum are expressed in terms of the collision cross section.
\end{abstract}

PACS numbers: 05.40.Jc, 03.65.Ca, 03.65.Sq, 05.60.Gg

The issue of quantum dissipation, and in particular of quantum Brownian motion, is a long-standing one, which has attracted physicists for decades (for general references see $[1,2])$ and still seems to be unsolved. Its relevance, however, is growing, especially in connection with decoherence and the relationship between classical and quantum description [3], a field which seems to be now within reach of experimental tests [4]. The classical understanding of the phenomenon is quite well established, relying on Langevin or Fokker-Planck equations obtained by considering a particle typically interacting with a bath of independent oscillators, so that most of the research has been influenced by these results, leading to research for a quantum analog or quantum generalization of these equations. The difficulty lies in the failure of a Hamiltonian description for such systems, so that a clear quantization prescription is missing, and one needs a thoroughly quantum mechanical approach. The most promising results come from the reduced description of a particle interacting with some type of reservoir, thus impinging on techniques and results of open quantum system theory. In this respect the property of complete positivity $(\mathrm{CP})$ has emerged as a very useful and stringent requirement in the study of subdynamics inside quantum mechanics [2,5]. The property of CP asks that the time evolution semigroup $\mathcal{U}(t)$ for the irreversible dynamics has the structure $\mathcal{U}(t) \varrho=$ $\sum_{\alpha} K_{\alpha}(t) \varrho K_{\alpha}^{\dagger}(t)\left(\sum_{\alpha} K_{\alpha}^{\dagger}(t) K_{\alpha}(t)=1\right)$ so that, in particular, positivity is preserved, and it originates from the formal requirement that coupling without interaction to an $n$-level system does not affect positivity. Indeed, CP appears somehow more natural if considered in the context of operations and quantum measurement in which it originally appeared in physics [6]. According to a famous paper by Lindblad [5], under suitable mathematical conditions the property of $\mathrm{CP}$ allows for the determination of the general structure of the generators of irreversible time evolutions, even though a thorough understanding of the physical limits of validity of this property is still on its way $[7,8]$, so that satisfaction of CP by itself does not ensure physically meaningful results, and the connection to realistic microphysical models is strongly desirable. In fact recent work has stressed the connection between $\mathrm{CP}$ of the time evolution and weak coupling, so that, for example, an uncorrelated statistical operator can consistently be considered as an initial condition, as well as coarse graining in time [9]. Most of the work carried out at a fundamental level regarding dissipative systems follows the Feynman-Vernon influence functional formalism (see $[1,10]$, and references therein) first used by Caldeira and Leggett [11], who have derived a standard model of quantum Brownian motion, which, however, has the drawback that it does not ensure the positivity of the statistical operator [12]. This shortcoming has been cured in various ways $[10,13]$, and within the independent oscillator model also a positive though not $\mathrm{CP}$ time evolution has recently been obtained [14]. On the side of CP time evolutions, very little has been done at a fundamental level, thus also leading to the conjecture that $\mathrm{CP}$ dissipative evolutions could not be obtained from first principles [15], while most approaches are either axiomatic or phenomenological [16], so that few insights about what type of physical systems might consistently be described by the Lindblad-type master equations can be gained.

In this Letter we give a derivation of $\mathrm{CP}$ quantum Brownian evolution based on some recent work on the description of coherent and incoherent particle-matter interaction [17], which has already been successfully applied to neutron optics [18]. This work relies on the introduction of a time scale for the description of the subdynamics of slowly varying degrees of freedom in the Heisenberg picture, in this specific case the heavy particle with respect to the background of thermal particles. The interaction is in terms of two-particle collisions described by the full $T$ matrix, and the structure ensuring $\mathrm{CP}$ naturally arises from the resolvent identity of scattering theory.

Derivation and structure of the master equation. - Let $H=H_{0}+H_{m}+V$ be the Hamiltonian of the whole confined system in second quantization, $H_{0}=\sum_{h} E_{h} a_{h}^{\dagger} a_{h}$ describing the particle (either a fermion or a boson in the state $u_{h}, H_{m}$ matter, and $V$ their mutual interaction. We intend to describe a single particle, thus considering for the total system the statistical operator $\varrho=\sum_{k h} a_{k}^{\dagger} \varrho^{m} a_{h} \varrho_{k h}$, where $\varrho^{m}$ describes matter, $\varrho_{k h}$ is a positive matrix with trace one, and we have $a_{h} \varrho^{m}=0, \varrho^{m} a_{h}^{\dagger}=0, \forall h$. In 
order to consider the subdynamics of the microsystem we exploit the following reduction formula, which connects the expectations of operators in the total Fock space $\mathcal{H}_{F}$ with those of operators in the one-particle Hilbert space $\mathcal{H}^{(1)}$.

$$
\operatorname{Tr}_{\mathcal{H}_{F}}(A \varrho)=\sum_{h, k} A_{h k} \varrho_{k h}=\operatorname{Tr}_{\mathcal{H}^{(1)}}(\hat{\mathrm{A}} \hat{\varrho})
$$

$$
\frac{\Delta_{\tau} \varrho_{k h}(t)}{\tau}=\frac{1}{\tau}\left[\varrho_{k h}(t+\tau)-\varrho_{k h}(t)\right]=\frac{1}{\tau}\left[\operatorname{Tr}_{\mathcal{H}_{F}}\left(a_{h}^{\dagger} a_{k} e^{-(i / \hbar) H \tau} \varrho(t) e^{(i / \hbar) H \tau}\right)-\varrho_{k h}(t)\right] .
$$

By exploiting the cyclic invariance of the trace, we are led to consider $H$-picture operators $a_{h}^{\dagger}(\tau) a_{k}(\tau)$, to be evaluated on the given time scale using a superoperator formalism, so that, e.g., $\mathcal{H}=\frac{i}{\hbar}[H, \cdot]$, using the integral representation

$$
a_{k}(\tau)=e^{\mathcal{H} \tau} a_{k}=\int_{-i \infty+\eta}^{+i \infty+\eta} \frac{d z}{2 \pi i} e^{z \tau}(z-\mathcal{H})^{-1} a_{k} .
$$

Introducing the superoperator $\mathcal{T}(z) \equiv \mathcal{V}+\mathcal{V}(z-$ $\mathcal{H})^{-1} \mathcal{V}$, which is the analog of the $T$ matrix, we have

$$
\begin{aligned}
(z-\mathcal{H})^{-1}= & \left(z-\mathcal{H}_{0}\right)^{-1} \\
& +\left(z-\mathcal{H}_{0}\right)^{-1} \mathcal{T}(z)\left(z-\mathcal{H}_{0}\right)^{-1},
\end{aligned}
$$

so that in the considered structure $a_{h}^{\dagger}(\tau) a_{k}(\tau)$, bilinear in the field operators, the emergence of a typically incoherent term having the CP structure $K_{\alpha} \varrho K_{\alpha}^{\dagger}$ bilinear in the $T$ matrix naturally appears, thus confirming recent phenomenological approaches $[19,20]$. Using the fact that $\left[H, \sum_{h} a_{h}^{\dagger} a_{h}\right]=0$ (the interaction potential being bilinear in the particle field operators), the restriction of $\mathcal{T}(z) a_{k}$ to the case of a single particle may be generally written $i \hbar \mathcal{T}(z) a_{k}=\sum_{h} T_{h}^{k}(i \hbar z) a_{h}$, where $T_{h}^{k}(z)$ is an operator in the Fock space of the macrosystem only. This matrix, which according to the introduction of the time scale should exhibit a slow energy dependence, plays a central role, accounting for the peculiarities of the interaction between the particle and the considered medium. The master equation we finally obtain has the following Lindblad form:

$$
\begin{aligned}
\frac{d \hat{\varrho}}{d \tau}= & -\frac{i}{\hbar}[\hat{\mathrm{H}}, \hat{\varrho}] \\
& +\frac{1}{\hbar} \sum_{\lambda, \xi}\left[\hat{\mathrm{L}}_{\lambda \xi} \hat{\varrho} \hat{\mathrm{L}} \hat{\mathrm{C}}_{\lambda \xi}^{\dagger}-\frac{1}{2}\left\{\hat{\mathrm{L}}_{\lambda \xi}^{\dagger} \hat{\mathrm{L}}_{\lambda \xi}, \hat{\varrho}\right\}\right],
\end{aligned}
$$

where $\hat{\mathrm{H}}=\hat{\mathrm{H}}_{0}+\hat{\mathrm{V}}$ and $\hat{\mathrm{V}}=\frac{1}{2}\left(\hat{\mathrm{Q}}+\hat{\mathrm{Q}}^{\dagger}\right)$ with

$$
\begin{aligned}
\langle k|\hat{Q}| h\rangle & =\operatorname{Tr}_{\mathcal{H}_{F}}\left[T_{h}^{k}\left(E_{k}+i \varepsilon\right) \varrho^{m}\right] \\
\left\langle k\left|\hat{\mathrm{L}}_{\lambda \xi}\right| h\right\rangle & =\sqrt{2 \varepsilon \pi_{\xi}} \frac{\left\langle\lambda\left|T_{h}^{k}\left(E_{k}+i \varepsilon\right)\right| \xi\right\rangle}{E_{k}+E_{\lambda}-E_{h}-E_{\xi}-i \varepsilon},
\end{aligned}
$$
kernel, and therefore

$$
T_{h}^{k}(z)=\sum_{\eta \mu} \delta_{p_{\eta}+p_{k}, p_{h}+p_{\mu}} b_{\eta}^{\dagger} \tilde{t}\left(z,\left|\boldsymbol{p}_{\mu}-\boldsymbol{p}_{\eta}\right|\right) b_{\mu} .
$$

where $A$ has the typical structure $A=\sum_{h, k} a_{h}^{\dagger} A_{h k} a_{k}=$ $\sum_{h, k} a_{h}^{\dagger}\langle h|\hat{\mathrm{A}}| k\rangle a_{k}$. We intend to work on a time scale $\tau$ much longer than microphysical collision time, being interested only in the slow dynamics of the particle, and we shall therefore approximate the time derivative with the following coarse-grained one:

with $\varrho^{m}=\sum_{\xi} \pi_{\xi}|\xi\rangle\langle\xi|$ being the statistical operator describing matter at equilibrium, and $|\lambda\rangle,|\xi\rangle$ denoting eigenvectors of $H_{m}$ with eigenvalues $E_{\lambda}, E_{\xi}$, while $|k\rangle$, $|h\rangle$ denote eigenvectors of $H_{0}$ with eigenvalues $E_{k}, E_{h}$. A detailed derivation of this master equation is given in Refs. [17,18]. In considering many-particle systems, important corrections due to statistics of identical particles appear. A generalization of the formalism to cope with dilute many-particle systems, in which statistic effects have been accounted for, has been considered in Ref. [21].

Completely positive quantum Brownian motion.Following [18] we will make the general Ansatz $T_{h}^{k}(z)=\int d^{3} \boldsymbol{x} \int d^{3} \boldsymbol{y} \psi^{\dagger}(\boldsymbol{x}) u_{k}^{*}(\boldsymbol{y}) t(z, \boldsymbol{x}-\boldsymbol{y}) u_{h}(\boldsymbol{y}) \psi(\boldsymbol{x})$, where we have supposed translation invariance in the interaction kernel, and $\psi^{\dagger}, \psi$ denote field operators for the macrosystem. Introducing creation and destruction operators $b^{\dagger}, b$ in the Fock space of the macrosystem, we may write $T_{h}^{k}(z)=\sum_{\eta \mu} b_{\eta}^{\dagger} T_{k \eta h \mu}(z) b_{\mu}$. Being interested in local dissipation effects we may safely suppose that, at least far away from the boundaries, the system is homogeneous so as to use as quantum numbers momentum eigenvalues, thus obtaining $T_{k \eta h \mu}(z)=\delta_{p_{\eta}+p_{k}, p_{h}+p_{\mu}} \tilde{t}\left(z,\left|p_{\mu}-p_{\eta}\right|\right)$, depending on the Fourier transform of the interaction

We may now insert this expression in (1) to evaluate the different contributions, starting from the last, typically incoherent term. In doing this we consider the medium as composed of free gas particles, so that the energy eigenstates $|\lambda\rangle,|\xi\rangle$ of $\varrho^{m}$ may be obtained by the repeated action of $b_{l}^{\dagger}$ on the vacuum, and we can write $|\lambda\rangle=\left|\left\{n_{l}^{\lambda}\right\}\right\rangle$, $l$ labeling the different momenta. We therefore simply have $\left\langle\lambda\left|b_{\eta}^{\dagger} b_{\mu}\right| \lambda\right\rangle=\delta_{\eta, \mu} n_{\mu}^{\lambda}$ and, taking the slow energy dependence of $\tilde{t}$ into account, the contributions with $\lambda=$ $\xi$ cancel out in the master equation and we need only consider the primed sum for $\lambda \neq \xi$. Using, for $\lambda \neq$ $\xi,\left\langle\lambda\left|b_{\eta}^{\dagger} b_{\mu}\right| \xi\right\rangle=\left(\prod_{\nu \neq \mu, \eta} \delta_{n_{\nu}^{\lambda}, n_{\nu}^{\xi}}\right) \delta_{\left(n_{\eta}^{\lambda}-1\right), n_{\eta}^{\xi}} \delta_{n_{\mu}^{\lambda},\left(n_{\mu}^{\xi}-1\right)}(1-$ $\left.\delta_{\eta, \mu}\right) \sqrt{n_{\mu}^{\xi}} \sqrt{n_{\eta}^{\lambda}}$, we come to, setting $\boldsymbol{Q}_{\mu \eta} \equiv \boldsymbol{p}_{\mu}-\boldsymbol{p}_{\eta}$,

$$
\begin{aligned}
\frac{1}{\hbar} \sum_{\lambda, \xi} \hat{\mathrm{L}}_{\lambda \xi} \hat{\varrho} \hat{\mathrm{L}}_{\lambda \xi}^{\dagger}= & \frac{2 \varepsilon}{\hbar} \sum_{p p^{\prime}} \sum_{\eta \mu}^{\prime}\left\langle n_{\mu}\left(1 \pm n_{\eta}\right)\right\rangle \frac{\tilde{t}\left(\left[\boldsymbol{p}+\boldsymbol{Q}_{\mu \eta}\right]^{2} / 2 M+i \varepsilon, Q_{\mu \eta}\right)}{\frac{\boldsymbol{p}_{\mu}^{2}}{2 m}-\frac{\left(\boldsymbol{p}_{\mu}-\boldsymbol{Q}_{\mu \eta}\right)^{2}}{2 m}+\frac{\boldsymbol{p}^{2}}{2 M}-\frac{\left(\boldsymbol{p}+\boldsymbol{Q}_{\mu \eta}\right)^{2}}{2 M}+i \varepsilon} \\
& \times e^{(i / \hbar) \boldsymbol{Q}_{\mu \eta} \cdot \hat{\mathrm{x}}}|\boldsymbol{p}\rangle\left\langle\boldsymbol{p}|\hat{\varrho}| \boldsymbol{p}^{\prime}\right\rangle\left\langle\boldsymbol{p}^{\prime}\right| e^{-(i / \hbar) \boldsymbol{Q}_{\mu \eta} \cdot \hat{\mathrm{x}}} \frac{\tilde{t}^{*}\left(\left[\boldsymbol{p}^{\prime}+\boldsymbol{Q}_{\mu \eta}\right]^{2} / 2 M+i \varepsilon, Q_{\mu \eta}\right)}{\frac{\boldsymbol{p}_{\mu}^{2}}{2 m}-\frac{\left(\boldsymbol{p}_{\mu}-\boldsymbol{Q}_{\mu \eta}\right)^{2}}{2 m}+\frac{\boldsymbol{p}^{\prime 2}}{2 M}-\frac{\left(\boldsymbol{p}^{\prime}+\boldsymbol{Q}_{\mu \eta}\right)^{2}}{2 M}-i \varepsilon},
\end{aligned}
$$


where $M$ denotes the mass of the Brownian particle, whose position operator is $\hat{\mathrm{X}}$, while $m$ is the mass of the gas particles. The Brownian particle is immersed in a nondegenerate gas, so that $\left\langle n_{\mu}\left(1 \pm n_{\eta}\right)\right\rangle=\left\langle n_{\mu}\right\rangle\left(1 \pm\left\langle n_{\eta}\right\rangle\right) \approx\left\langle n_{\mu}\right\rangle$. Considering now the quasidiagonality of the density ma- trix, linked to its slow variability, we substitute in the $T$ matrix and the denominators $\boldsymbol{p}, \boldsymbol{p}^{\prime}$ with the symmetric expression $\frac{1}{2}\left(\boldsymbol{p}+\boldsymbol{p}^{\prime}\right)$; furthermore, we use the variables $\boldsymbol{p}_{\mu}, \boldsymbol{q} \equiv \boldsymbol{Q}_{\mu \eta}$, and put into evidence the ratio $\alpha=m / M$ between the masses, thus coming to

$$
\begin{aligned}
\frac{4 \pi m}{\hbar} \sum_{p p^{\prime}} \sum_{q}^{\prime} & \frac{1}{q}\left|\tilde{t}\left(\left[\frac{\boldsymbol{p}+\boldsymbol{p}^{\prime}}{2}+\boldsymbol{q}\right]^{2} / 2 M+i \varepsilon, q\right)\right|^{2} \sum_{\mu}\left\langle n_{\mu}\right\rangle \delta\left[(1+\alpha) q+\alpha\left(\boldsymbol{p}+\boldsymbol{p}^{\prime}\right) \cdot \frac{\boldsymbol{q}}{q}-2 \boldsymbol{p}_{\mu} \cdot \frac{\boldsymbol{q}}{q}\right] \\
& \times e^{(i / \hbar) \boldsymbol{q} \cdot \hat{\mathrm{x}}}|\boldsymbol{p}\rangle\left\langle\boldsymbol{p}|\hat{\varrho}| \boldsymbol{p}^{\prime}\right\rangle\left\langle\boldsymbol{p}^{\prime}\right| e^{-(i / \hbar) \boldsymbol{q} \cdot \hat{\mathrm{x}}} .
\end{aligned}
$$

The anticommutator term can be treated in an analogous way, so that the final expression for the dissipative contributions in (1) becomes, neglecting for simplicity the slow energy dependence of the $T$ matrix,

$$
\begin{aligned}
\frac{4 \pi m}{\hbar} \sum_{q} \frac{|\tilde{t}(q)|^{2}}{q} \sum_{\mu}\left\langle n_{\mu}\right\rangle\{ & \sum_{p p^{\prime}} \delta\left[(1+\alpha) q+\alpha\left(\boldsymbol{p}+\boldsymbol{p}^{\prime}\right) \cdot \frac{\boldsymbol{q}}{q}-2 \boldsymbol{p}_{\mu} \cdot \frac{\boldsymbol{q}}{q}\right] e^{(i / \hbar) \boldsymbol{q} \cdot \hat{x}}|\boldsymbol{p}\rangle\left\langle\boldsymbol{p}|\hat{\varrho}| \boldsymbol{p}^{\prime}\right\rangle\left\langle\boldsymbol{p}^{\prime}\right| e^{-(i / \hbar) \boldsymbol{q} \cdot \hat{x}} \\
& \left.-\frac{1}{2} \sum_{p} \delta\left[(1+\alpha) q+2 \alpha \boldsymbol{p} \cdot \frac{\boldsymbol{q}}{q}-2 \boldsymbol{p}_{\mu} \cdot \frac{\boldsymbol{q}}{q}\right]\{|\boldsymbol{p}\rangle\langle\boldsymbol{p}|, \hat{\varrho}\}\right\} .
\end{aligned}
$$

We can now go over to the continuum limit in $\boldsymbol{p}_{\mu}$ and $\boldsymbol{q}$, evaluating the integral with respect to $\boldsymbol{p}_{\mu}$ in the case of a Boltzmann gas, using $\left\langle n_{\mu}\right\rangle=n \lambda_{m}^{3} \exp \left[-\beta\left(\boldsymbol{p}_{\mu}^{2} / 2 m\right)\right], \lambda_{m}$ being the thermal wavelength of the gas particles, $n$ their density, and $\beta=1 /\left(k_{\mathrm{B}} T\right)$ giving the temperature dependence. We thus obtain, in the Brownian limit $\alpha \ll 1$,

$$
\frac{4 \pi^{2} m^{2}}{\beta \hbar} n \lambda_{m}^{3} \int d^{3} \boldsymbol{q} \frac{|\tilde{t}(q)|^{2}}{q} e^{-(\beta / 8 m) q^{2}}\left[e^{(i / \hbar) \boldsymbol{q} \cdot \hat{x}} e^{-(\beta / 4 M) \boldsymbol{q} \cdot \hat{\mathrm{p}}} \hat{\varrho} e^{-(\beta / 4 M) q \cdot \hat{\mathrm{p}}} e^{-(i / \hbar) \boldsymbol{q} \cdot \hat{\mathrm{x}}}-\frac{1}{2}\left\{e^{-(\beta / 2 M) \boldsymbol{q} \cdot \hat{\mathrm{p}}}, \hat{\varrho}\right\}\right] .
$$

To get to the master equation describing quantum dissipation we want to extract the temperature dependence of this expression, in the limit of small momentum transfer $q$. We therefore expand the exponential operators up to second order in $\boldsymbol{q}$, which is also equivalent to keeping terms at most bilinear in the operators $\hat{x}$ and $\hat{p}$. Because of symmetry properties of the coefficients, only terms bilinear in $\boldsymbol{q}$ and of the form $\boldsymbol{q}_{i}^{2}$ ( $i$ denoting Cartesian coordinates) need be retained, so that we have

$$
-\frac{2 \pi^{2} m^{2}}{\beta \hbar} n \lambda_{m}^{3} \int d^{3} \boldsymbol{q} \frac{|\tilde{t}(q)|^{2}}{q} e^{-(\beta / 8 m) q^{2}} \sum_{i=1}^{3} \boldsymbol{q}_{i}^{2}\left\{\frac{1}{\hbar^{2}}\left[\hat{\mathrm{x}}_{i},\left[\hat{\mathrm{x}}_{i}, \hat{\varrho}\right]\right]+\frac{\beta^{2}}{16 M^{2}}\left[\hat{\mathrm{p}}_{i},\left[\hat{\mathrm{p}}_{i}, \hat{\varrho}\right]\right]+\frac{i}{\hbar} \frac{\beta}{2 M}\left[\hat{\mathrm{x}}_{i},\left\{\hat{\mathrm{p}}_{i}, \hat{\varrho}\right\}\right]\right\} .
$$

Let us note that in the derivation important cancellations and compensations arise between the terms coming from the anticommutator and incoherent part of (1), necessary in order to obtain the final structure, thus confirming the fact that in quantum theory we cannot have separate friction and diffusion terms [22]. Decisive for the determination of the final structure of the equation is also the Brownian limit $\alpha=m / M \ll 1$. Supposing, without loss of generality, the medium to be isotropic, so that $\boldsymbol{q}_{i}^{2}=\frac{1}{3} q^{2}$, we can define the following coefficient:

$$
D_{p p}=\frac{2}{3} \frac{\pi^{2} m^{2}}{\beta \hbar} n \lambda_{m}^{3} \int d^{3} \boldsymbol{q}|\tilde{t}(q)|^{2} q e^{-(\beta / 8 m) q^{2}},
$$

depending on the collision cross section through the $T$ matrix, and obtain the compact expression

$$
\begin{aligned}
\sum_{i=1}^{3}\{ & \frac{D_{p p}}{\hbar^{2}}\left[\hat{\mathrm{x}}_{i},\left[\hat{\mathrm{x}}_{i}, \hat{\varrho}\right]\right]+\frac{D_{q q}}{\hbar^{2}}\left[\hat{\mathrm{p}}_{i},\left[\hat{\mathrm{p}}_{i}, \hat{\varrho}\right]\right] \\
& \left.+\frac{i}{\hbar} \gamma\left[\hat{\mathrm{x}}_{i},\left\{\hat{\mathrm{p}}_{i}, \hat{\varrho}\right\}\right]\right\},
\end{aligned}
$$

where $D_{q q}=(\beta \hbar / 4 M)^{2} D_{p p} \quad$ and $\quad \gamma=(\beta / 2 M) D_{p p}$. Exploiting (2) for the $T$ matrix we simply obtain, for the potential term in the continuum limit, $\hat{\mathrm{V}}=$ $-n \frac{2 \pi \hbar^{2}}{m} \int d^{3} \boldsymbol{p}|\boldsymbol{p}\rangle\langle\boldsymbol{p}| \operatorname{Re} f\left(E_{p}, \theta=0\right), n$ being the density of the gas particles, so that it essentially depends on the forward scattering amplitude $f\left(E_{p}, \theta=0\right)$ as expected, and vanishes if the latter does not depend on energy. The complete master equation then becomes

$$
\begin{aligned}
\frac{d \hat{\varrho}}{d t}= & -\frac{i}{\hbar}\left[\hat{\mathrm{H}}_{0}+\hat{\mathrm{V}}, \hat{\varrho}\right]-\frac{D_{p p}}{\hbar^{2}} \sum_{i=1}^{3}\left[\hat{\mathrm{x}}_{i},\left[\hat{\mathrm{x}}_{i}, \hat{\varrho}\right]\right] \\
& -\frac{D_{q q}}{\hbar^{2}} \sum_{i=1}^{3}\left[\hat{\mathrm{p}}_{i},\left[\hat{\mathrm{p}}_{i}, \hat{\varrho}\right]\right]-\frac{i}{\hbar} \gamma \sum_{i=1}^{3}\left[\hat{\mathrm{x}}_{i},\left\{\hat{\mathrm{p}}_{i}, \hat{\varrho}\right\}\right] .
\end{aligned}
$$

This is the main result of this Letter: a CP time evolution for a quantum Brownian particle derived at a fundamental level, using a different, new approach with respect to the usual independent oscillator model. The equation obtained is translationally invariant and has the correct Lindblad form [22,23]. In particular, the requirement for $\mathrm{CP}$ amounts to checking that $D_{p p} D_{q q} \geq \hbar^{2} \gamma^{2} / 4$, which in our case is verified with the equal sign, thus uniquely determining the different coefficients in a structure such as (4) apart from an overall multiplying factor. Let us note that the requirement of a stationary thermal equilibrium solution determines only the ratio between $D_{p p}$ and $\gamma$, and also CP simply indicates that the coefficient $D_{q q}$ should be different from zero and within some range, without 
actually fixing it. This explains the wide variety of different contributions that have been added to the Caldeira equation to make it preserve positive definiteness. The fact that $D_{p p} D_{q q}=\hbar^{2} \gamma^{2} / 4$ has, as a consequence, the following interesting distinctive feature: in order to write (4) in a manifest Lindblad form, only one generator for each Cartesian direction has to be introduced, instead of two. In fact, using the thermal wavelength $\lambda_{M}=\sqrt{\hbar^{2} / M k T}$ associated with the Brownian particle and defining the operators $\hat{\mathrm{a}}_{i}=\frac{\sqrt{2}}{\lambda_{M}}\left(\hat{\mathrm{x}}_{i}+\frac{i}{\hbar} \frac{\lambda_{M}^{2}}{4} \hat{\mathrm{p}}_{i}\right)$, satisfying $\left[\hat{\mathrm{a}}_{i}, \hat{\mathrm{a}}_{j}^{\dagger}\right]=\delta_{i j}$, we can rewrite (4) in the form

$$
\begin{aligned}
\frac{d \hat{\varrho}}{d t}= & -\frac{i}{\hbar}\left[\hat{\mathrm{H}}_{0}+\hat{\mathrm{V}}, \hat{\varrho}\right]-\frac{D_{p p}}{\hbar^{2}} \frac{\lambda_{M}^{2}}{4} \sum_{i=1}^{3} \frac{i}{\hbar}\left[\left\{\hat{\mathrm{x}}_{i}, \hat{\mathrm{p}}_{i}\right\}, \hat{\varrho}\right] \\
& +\frac{D_{p p}}{\hbar^{2}} \lambda_{M}^{2} \sum_{i=1}^{3}\left[\hat{\mathrm{a}}_{i} \hat{\varrho} \hat{\mathrm{a}}_{i}^{\dagger}-\frac{1}{2}\left\{\hat{\mathrm{a}}_{i}^{\dagger} \hat{\mathrm{a}}_{i}, \hat{\varrho}\right\}\right]
\end{aligned}
$$

This makes an important qualitative difference with a more phenomenological model derived by Diósi [20], also linked to the fact that he obtains an equation with the asymmetric expression $\boldsymbol{p}_{\mu}$ instead of the momentum transfer $\boldsymbol{q}$. This connection between the number of generators and relationships among the coefficients in a master equation of the form (4) has not been stressed in the literature, even though it provides an important qualitative feature, helpful in providing clear-cut distinctions. In this spirit our work also sheds some light on the recent phenomenological work of Gao [24] and the subsequent following debate $[25,26]$. Gao works from the very beginning with a single generator $V=\mu \hat{\mathrm{x}}+i \nu \hat{\mathrm{p}}$ and this automatically leads him to obtain a generalized Caldeira equation with $D_{q q}=\gamma /\left(8 M k_{\mathrm{B}} T\right)$, so that $D_{p p} D_{q q}=\hbar^{2} \gamma^{2} / 4$ is verified. This explains the difference from the diffusion coefficient $D_{q q}=\gamma /\left(6 M k_{\mathrm{B}} T\right)$ found in Ref. [13]. Despite the fact that the coefficients in the master equation with which Gao starts are actually completely fixed by the requirement of thermal equilibrium and his choice of a single generator, our work provides some fundamental evidence in favor of this structure, giving through (3) the quantitative estimate $\gamma=(\beta / 2 M) D_{p p}$ for the relaxation coefficient. The heavily criticized [25] Hamiltonian term $-\frac{\gamma}{2}\{\hat{x}, \hat{p}\}$ which Gao obtains, however, does not appear in (4), so that no fictitious counterterm is necessary: Its appearance in rewriting (4) in the form (5) clarifies why the initial choice of Gao led to this trouble.

We have thus obtained a new fundamental derivation of $\mathrm{CP}$ dissipative evolution, driven by collisions with the environment, with temperature dependent friction and diffusion coefficients expressed in terms of physical quantities such as the collision cross section. The associated master equation has the peculiarity of being expressible in Lindblad form with only a single generator for each Cartesian direction, thus giving some evidence in favor of a recent phenomenological model [24], though being deprived of its unphysical features $[25,26]$. The underlying calculations, even though recovering a single-particle description by tracing over matter, are rooted in a second quantization formalism conceived for the description of a subset of reduced degrees of freedom slowly varying on a given time scale $[17,21]$.

I am very indebted to Professor L. Lanz who followed the whole work, and I would like to thank Professor A. Barchielli and Professor O. Melsheimer for useful discussions. This work was supported by the Alexander von Humboldt-Stiftung.

[1] H. Dekker, Phys. Rep. 80, 1 (1981); H. Grabert, P. Schramm, and G.-L. Ingold, Phys. Rep. 168, 115 (1988).

[2] R. Alicki and K. Lendi, Lecture Notes in Physics (Springer, Berlin, 1987), Vol. 286.

[3] D. Giulini et al., Decoherence and the Appearance of a Classical World in Quantum Theory (Springer, Berlin, 1996).

[4] M. Brune et al., Phys. Rev. Lett. 77, 4887 (1996).

[5] G. Lindblad, Commun. Math. Phys. 48, 119 (1976).

[6] K. Kraus, Lecture Notes in Physics (Springer, Berlin, 1983), Vol. 190; K.-E. Hellwig, Int. J. Theor. Phys. 34, 1467 (1995).

[7] R. C. de Berrêdo et al., Phys. Scr. 57, 533 (1998).

[8] P. Pechukas, Phys. Rev. Lett. 73, 1060 (1994); 75, 3021 (1995); R. Alicki, ibid. 75, 3020 (1995).

[9] A. Royer, Phys. Rev. Lett. 77, 3272 (1996); A. Suarez, R. Silbey, and I. Oppenheim, J. Chem. Phys. 97, 5101 (1992).

[10] B. L. Hu, J. P. Paz, and Y. Zhang, Phys. Rev. D 45, 2843 (1992).

[11] A. O. Caldeira and A.J. Leggett, Physica (Amsterdam) 121A, 587 (1983).

[12] V. Ambegaokar, Ber. Bunsen-Ges. Phys. Chem. 95, 400 (1991).

[13] L. Diósi, Physica (Amsterdam) 199A, 517 (1993); Europhys. Lett. 22, 1 (1993).

[14] A. Tameshtit and J.E. Sipe, Phys. Rev. Lett. 77, 2600 (1996).

[15] W. J. Munro and C. W. Gardiner, Phys. Rev. A 53, 2633 (1996).

[16] H. Dekker, Phys. Rev. A 16, 2126 (1977); A. Săndulescu and H. Scutaru, Ann. Phys. (N.Y.) 173, 277 (1987); M. R. Gallis, Phys. Rev. A 48, 1028 (1993).

[17] L. Lanz and B. Vacchini, Int. J. Theor. Phys. 36, 67 (1997).

[18] L. Lanz and B. Vacchini, Phys. Rev. A 56, 4826 (1997).

[19] S. Stegholm, Phys. Scr. 47, 724 (1993).

[20] L. Diósi, Europhys. Lett. 30, 63 (1995).

[21] L. Lanz and B. Vacchini, Int. J. Theor. Phys. 37, 545 (1998); L. Lanz and O. Melsheimer, in Lecture Notes in Physics, edited by A. Bohm, H.-D. Doebner, and P. Kielanowski (Springer, Berlin, 1998), Vol. 504, p. 345.

[22] G. Lindblad, Rep. Math. Phys. 10, 393 (1976).

[23] A. Barchielli, Nuovo Cimento 74B, 113 (1983).

[24] S. Gao, Phys. Rev. Lett. 79, 3101 (1997).

[25] H. W. Wiseman and W. J. Munro, Phys. Rev. Lett. 80, 5702 (1998); S. Gao, ibid. 80, 5703 (1998).

[26] G. W. Ford and R. F. O'Connell, Phys. Rev. Lett. 82, 3376 (1999); S. Gao, ibid. 82, 3377 (1999). 